\title{
Axillary brachial plexus block-an underused technique in the accident and emergency department
}

\author{
C A Mackay, D F Bowden
}

\begin{abstract}
Objective-To compare axillary brachial plexus block and Bier's block as methods of providing upper limb anaesthesia.

Methods-Axillary brachial plexus or Bier's blocks were performed on all patients requiring upper limb anaesthesia in a three month period. For Bier's block, a single cuff tourniquet and $3 \mathrm{mg} / \mathrm{kg} 0.5 \%$ prilocaine were used. For axillary plexus block, $40 \mathrm{ml} 1 \%$ lignocaine with adrenaline (1:200 000) were used, given by perivascular or transarterial technique. Prospective analysis was made of time to complete limb anaesthesia, type of procedure performed, and duration of limb anaesthesia. Patient perception of analgesia and satisfaction with the method of anaesthesia was assessed using a 10 point visual analogue scale.
\end{abstract}

Results-75 patients underwent procedures requiring upper limb anaesthesia; 39 received axillary plexus block and 36 Bier's block. $72 \%$ of Bier's blocks and $77 \%$ of axillary plexus blocks provided complete anaesthesia without the need for supplemental analgesia. The median time to onset of anaesthesia was $10 \mathrm{~min}$ for Bier's block and $32.5 \mathrm{~min}$ for axillary block $(P<0.001)$. The median duration of anaesthesia was $15 \mathrm{~min}$ for Bier's block and 240 min for axillary block $(P<0.001)$. Mean scores for analgesia were 9.7 for axillary blocks and 8.8 for Bier's block $(P<0.001) .87 \%$ of the axillary block group were completely satisfied with the method of anaesthesia, compared with $56 \%$ of the Bier's block group.

Conclusions-Brachial plexus blocks are an alternative form of providing upper limb anaesthesia in the accident and emergency department. They are relatively simple to perform, well tolerated by patients, and have the advantage of providing prolonged analgesia without the need for additional medication. $(\Im$ Accid Emerg Med 1997;14:226-229)

Keywords: axillary brachial plexus block; intravenous regional anaesthesia; local anaesthetic; haematoma block

Correspondence to:

C A Mackay, senior registrar,

Accident and Emergency

Department, Walsall Manor

Hospital, Moat Road, Walsall WS2 9PS.
Several procedures carried out in the accident and emergency (A\&E) department, such as fracture manipulation and tendon repair, require upper limb anaesthesia. Traditionally this has been achieved using general anaesthesia, intravenous regional anaesthesia (Bier's block), haematoma block, or intravenous sedation, which all have their own associated problems. Axillary brachial plexus block is a well recognised method of achieving upper limb anaesthesia. However, its use in the $A \& E$ department is not common. This form of anaesthesia provides sensory and motor blockade of the limb in addition to sympathetic block to blood vessels, leading to a reduction in postoperative pain and oedema. We compare the use of this regional anaesthetic technique with the use of Bier's block in our A\&E department.

\section{Methods}

All patients aged 13 years or over requiring upper limb anaesthesia for a procedure in the A\&E department in the three month period 1 January 1996 to 31 March 1996 were eligible for inclusion. Exclusion criteria were sensitivity to local anaesthetic agents, sickle cell or Raynaud disease, or inability to give informed consent. Intravenous regional anaesthesia (Bier's block) had been routinely used to provide limb anaesthesia in the department. Therefore all members of staff familiar with this technique continued its use. Any patient attended to by one of the authors who required upper limb anaesthesia received an axillary brachial plexus block. No premedication was given and venous access was secured on the contralateral hand in all patients. Full resuscitation facilities were available at all times.

Patients undergoing Bier's block had a cannula inserted in the dorsum of the ipsilateral hand. The limb was elevated for three minutes to achieve exsanguination. A single cuff tourniquet was then inflated over wool padding to at least $50 \mathrm{~mm} \mathrm{Hg}$ above systolic blood pressure. After lowering the arm, prilocaine $0.5 \%$ was injected slowly into the indwelling cannula in a dose of $3 \mathrm{mg} / \mathrm{kg}$. The tourniquet cuff was not deflated after completion of the procedure until a minimum period of 20 minutes had elapsed following injection of local anaesthetic.

Patients undergoing axillary brachial plexus block were placed in the supine position with the arm externally rotated and abducted to 90 degrees. The axillary artery was identified by palpation and a 23 gauge needle inserted above the artery at an angle of 20 degrees to the skin and directed towards the apex of the axilla. A total volume of $40 \mathrm{ml}$ of $1 \%$ lignocaine with 
Table 1 Procedures carried out in the AEE department under axillary plexus or Bier's block

\begin{tabular}{lll}
\hline Procedure & $\begin{array}{l}\text { Bier's block } \\
\text { (number of patients) }\end{array}$ & $\begin{array}{l}\text { Axillary block } \\
\text { (number of patients) }\end{array}$ \\
\hline Colles fracture manipulation & 33 & 35 \\
Smith fracture manipulation & 3 & 2 \\
Metacarpal fracture manipulation & 0 & 1 \\
Extensor tendon repair & 0 & 1 \\
\hline
\end{tabular}

adrenaline (1:200 000) was injected. For patients weighing than $65 \mathrm{~kg}$, a dose of $0.6 \mathrm{ml} / \mathrm{kg}$ of local anaesthetic was used and diluted to a volume of $40 \mathrm{ml}$ using normal saline. An attempt was made to use the perivascular route in all cases. However, if blood was aspirated during the procedure, a transarterial block was used, that is, the needle was advanced through the artery until aspiration of blood no longer occurred and local anaesthetic was subsequently injected. Care was taken to ensure the needle remained outside the artery by repeatedly aspirating after every $5 \mathrm{ml}$ injection of anaesthetic. After completion of injection the arm was brought to the patient's side and firm pressure applied over the artery to prevent haematoma formation and to encourage proximal spread of local anaesthetic. Paraesthesia was not deliberately elicited at any stage during the procedure.

Sensory loss was assessed at five minute intervals with a 23 gauge needle using a scale 0-2 ( $0=$ no sensory loss, $1=$ loss of pinprick sensation, $2=$ loss of touch). For axillary nerve blocks sensory loss was assessed in the distribution of all peripheral nerves distal to the axillary nerve. A complete block was one in which surgical anaesthesia occurred. An incomplete block was classified as one in which pinprick but not touch was lost and which required supplemental analgesia. A failed block was one in which sensory blockade did not occur.

The time from injection of local anaesthetic to complete surgical anaesthesia in the limb was documented. Patients were instructed in the use of a 10 point visual analogue scale preoperatively and following completion of the procedure they were asked to evaluate the level of analgesia achieved and their general satisfaction with the method of anaesthesia. Before discharge, all patients were asked to document the time normal sensation returned to the limb. Patients were reviewed the following day

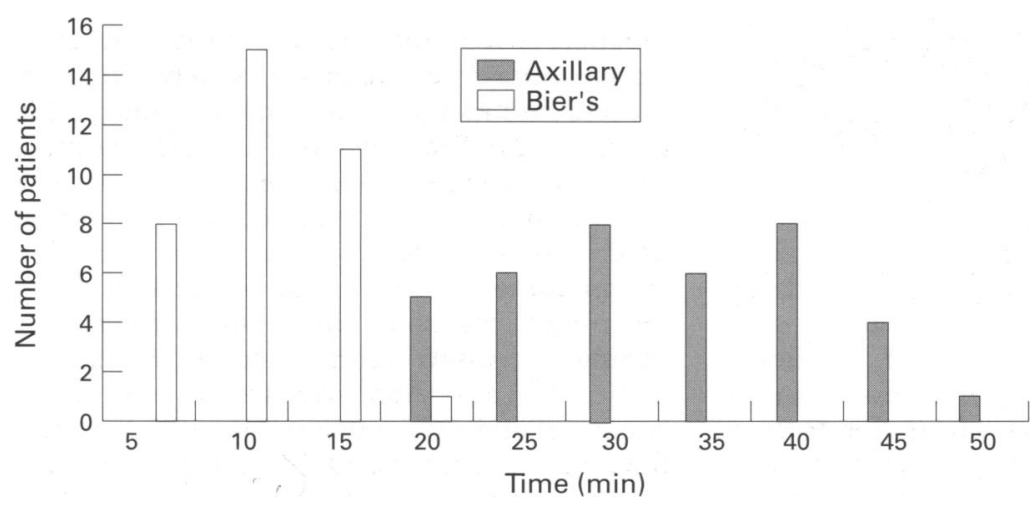

Figure 1 Time to onset of limb anaesthesia.

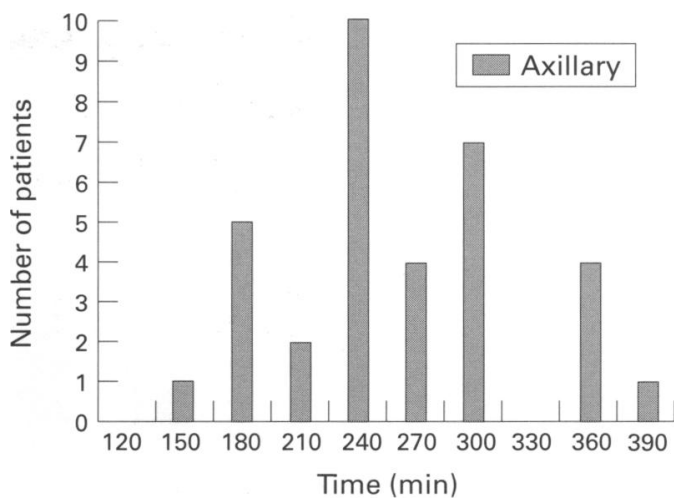

Figure 2 Duration of anaesthesia for axillary brachial plexus block.

to record this information and to assess any complications which may have arisen.

Statistical analysis was carried out using the KruskallWallis test for non-parametric data. A probability level of $<0.05$ was taken to indicate statistical significance.

\section{Results}

Seventy five patients were entered into the study. Thirty nine received axillary plexus blockade and thirty six Bier's block. The median age was 64 years (range 20-88) for axillary blocks and 70.5 years (range $40-93$ ) for Bier's blocks. Both groups were comparable in terms of sex and degree of fracture displacement.

Procedures carried out are documented in table 1 .

Twenty six patients $(72 \%)$ undergoing Bier's block experienced total sensory blockade and $10(28 \%)$ had incomplete anaesthesia, requiring additional analgesia. Thirty axillary brachial plexus blocks $(77 \%)$ were complete, eight (21\%) were incomplete, and one patient $(2 \%)$ had a failed block. All incomplete blocks in this group occurred in patients with Colles fractures, and in all cases the radial nerve territory was inadequately anaesthetised. No procedure was abandoned because of inadequate anaesthesia. One patient who had received an axillary block required remanipulation of the fracture following $x$ ray review. No additional analgesia was required for this.

Figure 1 shows time to complete limb anaesthesia in both groups. The median time for axillary block was 32.5 minutes and for Bier's block 10 minutes $(P<0.001)$.

Figures 2 and 3 show the duration of anaesthesia for each group. Three patients who had received brachial plexus blockade were unable to recall the time normal sensation returned to the limb. The median duration of anaesthesia for the remainder was 240 minutes, compared to 15 minutes for the Bier's block group $(\mathrm{P}<0.001)$.

Using visual analogue scales the mean analgesia score was 9.7 out of a possible maximum of 10 for patients receiving axillary plexus blocks, compared with 8.8 for patients undergoing Bier's block $(P<0.001)$. The lower scoring in the Bier's block group was mainly due to patients experiencing pain at the site of the tourniquet cuff. Using the same scale for 


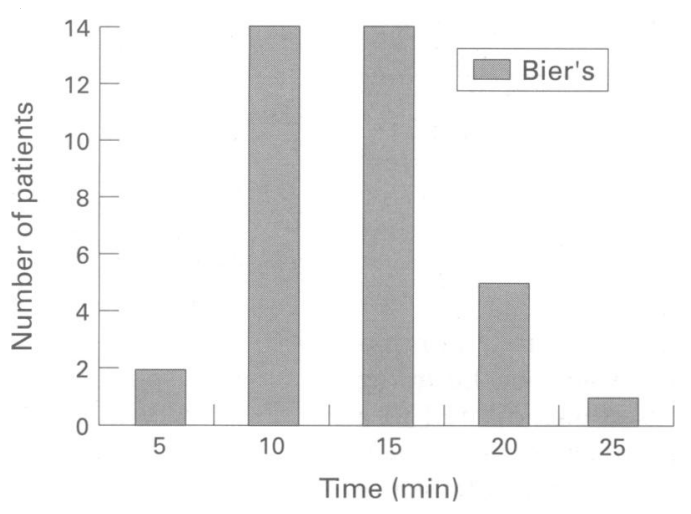

Figure 3 Duration of anaesthesia for Bier's block.

satisfaction with the method of anaesthesia, 33 patients $(87 \%)$ scored $10 / 10$ in the axillary block group compared with 20 patients (56\%) in the Bier's block group.

One patient undergoing axillary plexus block developed a sinus tachycardia associated with palpitations shortly after the injection of local anaesthetic. This settled spontaneously after 10 minutes. One patient complained of tinnitus and another of dizziness after deflation of the tourniquet cuff following Bier's block. These symptoms lasted less than five minutes and were not associated with any other features of local anaesthetic toxicity. No patient had any evidence of residual neurological deficit or axillary haematoma on review at 24 hours.

\section{Discussion}

A recent survey of United Kingdom A\&E departments showed that the majority use intravenous regional anaesthesia, haematoma block, or general anaesthesia as method of choice for manipulation of closed fractures of the distal radius. ${ }^{1}$ Only nine departments used regional blocks. Since the majority of patients undergoing such procedures are often elderly, almost invariably not fasted, and may have concomitant medical disorders, the use of general anaesthesia is often not ideal and also relies on the availability of an anaesthetist. Intravenous regional anaesthesia has been associated with intravascular leak of local anaesthetic due to tourniquet failure (leakage around an intact cuff or accidental deflation). ${ }^{23}$ Toxic levels of prilocaine have been recorded following fracture manipulation using Bier's block. ${ }^{4}$ Haematoma blocks are now widely used as a method of providing analgesia at the fracture site..$^{5}$ Although this has the advantage of being less time consuming than intravenous regional anaesthesia, analgesia is not always optimal $^{6}$ and toxic levels of lignocaine have been documented following fracture manipulation. ${ }^{7}$ The use of intravenous sedation and analgesia is preferred in some departments. Sedation alone provides no analgesia, which is unsatisfactory, and a combination of sedation and analgesia may render the patient sufficiently drowsy to be at risk of aspiration. Prolonged observation is also necessary in this group to ensure full recovery from the effects of these drugs. ${ }^{8}$

We have shown that axillary plexus block offers an alternative form of anaesthesia to the more traditionally preferred methods. However, its use in the $\mathrm{A} \& \mathrm{E}$ department is not common, presumably because of lack of familiarity with the technique.

The first brachial plexus block is thought to have been carried out by Halsted in 1884 . Over the years four routes of administration of local anaesthetic have been described, the interscalene, ${ }^{9}$ supraclavicular, ${ }^{10}$ infraclavicular, ${ }^{11}$ and axillary approaches. The supraclavicular approach has the risk of pneumothorax, and both supraclavicular and interscalene blocks may be associated with phrenic nerve paralysis and spread of local anaesthetic to block the vagus nerve and stellate ganglion. For these reasons the axillary approach has become increasingly popular.

Burnham described the axillary approach to brachial plexus blockade in $1958^{12}$ using a perivascular technique involving penetration of the axillary sheath above and below the axillary artery. This axillary perivascular approach was modified to a single injection technique by Winnie and Collins, ${ }^{10}$ and a transarterial approach was later described by Cockings et al in $1987 . .^{13}$ Most studies have shown a small complete failure rate, where it is assumed that the injection of local anaesthetic occurred outside the neurovascular bundle. Incomplete blockade is more common, with the axillary, musculocutaneous, and radial nerves most likely to be inadequately anaesthetised. ${ }^{14-17}$ No group has been able to duplicate the $99 \%$ success rate achieved by Cockings et al using the transarterial approach. ${ }^{13}$

One of the main theories for failure of the block was thought to be the presence of septa within the axillary sheath. ${ }^{18}$ Vester-Andersen $e t$ $a l^{19}$ and Partridge et $a l^{20}$ showed that these septa were incomplete and did not act as a barrier to local anaesthetic spread. A minimum volume of $40 \mathrm{ml}$ of anaesthetic appears necessary for successful block of the musculocutaneous nerve. ${ }^{14}$ Increasing the volume is associated with improved sensory blockade in the distribution of the axillary nerve $\mathrm{e}^{2122}$ but does not appear to have any effect on radial nerve blockade. Increasing the concentration of local anaesthetic used has a marked effect on motor but not sensory blockade. ${ }^{23}$

Our study has shown that axillary brachial plexus block provides adequate anaesthesia for upper limb procedures. The advantages of this technique are that insertion of the block is neither time consuming nor complicated, no sedation is required, and a prolonged postoperative period of analgesia is achieved. The success rate in achieving complete anaesthesia was similar to Bier's block. The main drawback would appear to be latency of onset of anaesthesia. Various techniques have been used in an attempt to reduce the time to onset of anaesthesia, the most successful being warming, ${ }^{24}$ carbonating, ${ }^{25}$ alkalinising, ${ }^{26}$ or adding potassium chloride ${ }^{27}$ to the local anaesthetic. In our $\mathrm{A} \& \mathrm{E}$ department, however, latency of onset was not seen as a disadvantage. Injection of local anaesthetic takes only a few minutes, leaving the operator free to attend to other patients in the department while waiting 
for anaesthesia to occur. For patients presenting with obviously deformed wrists which will require manipulation, we insert axillary blocks before the patient is sent for $x$ ray and the limb is often anaesthetic on the patient's return to the department, allowing immediate manipulation with no unnecessary patient delay.

With the exception of one case of transient sinus tachycardia, no other complications were noted. Most published studies have described the technique of axillary brachial plexus blockade using a blunt ended regional block needle which is not routinely available in most $A \& E$ departments. We did not encounter any evidence of neural or vascular damage when using a 23 gauge needle with caution, in keeping with the findings of Rouholamin and Harris who used the same technique for hand surgery without encountering any problems. ${ }^{28}$ The technique was well tolerated by all patients, achieving higher analgesia scores than Bier's block and with more patients expressing satisfaction with this method of anaesthesia than with intravenous regional anaesthesia. The number of patients requiring supplemental analgesia with Bier's block in this study was higher than might be expected. This may be accounted for by the fact that patients were not properly randomised to receive Bier's block or axillary nerve block, the technique used being dependent on the doctor treating the patient. Secondly the use of a double rather than single cuff may have reduced the incidence of cuff discomfort.

\section{CONCLUSIONS}

Axillary brachial plexus blockade offers an alternative approach to the more traditional methods for patients requiring upper limb anaesthesia in the $A \& E$ department. It is relatively simple to perform and provides satisfactory anaesthesia in most cases, with prolonged postoperative analgesia. The procedure is well tolerated by patients and is associated with few side effects. We believe that regional anaesthesia is underused in the A\&E department, and familiarity with this technique will extend its benefits to a wider patient population.

1 O'Sullivan I, Brooks S, Maryosh J. Is fasting necessary before prilocaine Bier's block? J Accid Emerg Med 1996;13:105-7.
2 Goold JE. Intravenous regional anaesthesia. Br J Hosp Med 1985; June:335-40.

3 Bartholomew K, Sloan JP. Prilocaine for Bier's block: how safe is safe? Arch Emerg Med 1990;7:189-95.

4 Quinton DN, Hughes J, Mace PFK, Aitkenhead AR. Prilocaine leakage during tourniquet inflation in intravenous regional anaesthesia: the influence of fracture manipulation. Injury 1988;19:333-5.

5 Case RD. Haematoma block-a safe method of reducing Colles' fractures. Injury $1985 ; 16: 469-70$.

6 Cobb AG, Houghton GR. Local anaesthetic infiltration versus Bier's block for Colles' fractures. BMJ 1985;291: 1683-4.

7 Quinton DN. Local anaesthetic toxicity of haematoma blocks in manipulation of Colles' fractures. Injury 1988;19: 239-40.

8 Armstrong P, Payne M, Brockway M. Anaesthesia and Colles' fractures. BMJ 1990;300:261.

9 Winnie AP. Interscalene brachial plexus blocks. Anesth Analg 1970;49:455-66.

10 Winnie AP, Collins VJ. The subclavian perivascular technique of brachial plexus anaesthesia. Anesthesiology 1964;25:353-63.

11 Raj PP, Montgomery SJ, Nettles D, Jenkins MT. Infraclavicular brachial plexus block: a new approach. Anesth Analg 1973;52:897-904.

12 Burnham BJ. Regional block of great nerves of the upper arm. Anesthesiology 1958;19:281.

13 Cockings E, Moore PL, Lewis RC. Transarterial brachial plexus blockade using high doses of $1.5 \%$ mepivicaine. Reg Anaesth 1987;12:159-64.

14 De Jong RH. Axillary blocks of the brachial plexus. Anesthesiology 1961;22:215-25.

15 Lanz E, Theiss D, Janovich DD. The extent of blockade following various techniques of brachial plexus block. Anesth lowing various techniqu

16 Youssef MS, Desgrand DA. Comparison of two methods of axillary brachial plexus anaesthesia. Br J Anaesth 1988;60: $841-4$

17 Brockway MS, Wildsmith JAW. Axillary brachial plexus block: method of choice? Br J Anaesth 1990;64:224-31.

18 Thompson GE, Rorie DK. Functional anatomy of the brachial plexus sheath. Anesthesiology 1983;59:117-22.

19 Vester-Andersen T, Broby-Johansen U, Bro-Rasmussen F Perivascular axillary block VI: the distribution of gelatine solution injected into the axillary neurovascular sheath of cadavers. Acta Anaesthesiol Scand 1986;30:18-22.

20 Partridge BL, Katz J, Benirschke K. Functional anatomy of the brachial plexus sheath: implications for anaesthesia. Anesthesiology 1987;66:743-7.

21 Vester-Andersen $T$, Husum B, Lindeburg $T$, Borrits $L$ Gothgen I. Perivascular axillary block IV: blockade following 40,50 or $60 \mathrm{mls}$ of mepivicaine $1 \%$ with adrenaline ing 40, 50 or $60 \mathrm{mls}$ of mepivicaine $1 \%$

22 Vester-Andersen T, Christiansen T, Sorensen M, KaalundJorgensen HO, Saujberg P, Schultz-Moller K. Perivascular axillary block II: influence of injected volume of loca anaesthetic on neural blockade. Acta Anaesthesiol Scand 1983;27:95-8.

23 Vester-Andersen T, Eriksen C, Christiansen C. Perivascular axillary block III: blockade following $40 \mathrm{mls} 0.5 \%, 1.0 \%$ or $1.5 \%$ mepivicaine with adrenaline. Acta Anaesthesiol Scand 1984;28:95-8.

24 Heath PJ, Brownlie GS, Herrick MJ. Latency of brachial plexus block. Anesthesia 1990;45:297-301.

25 McClure JH, Scott DB. Comparison of bupivicaine hydrochloride and carbonated bupivicaine in brachial heco blo by the interscalene technique. $\mathrm{Br}$ J Anaesth 1981;53:523-6.

26 Hilgier M. Alkalinisation of bupivicaine for brachial plexus block. Reg Anesth 1985;10:59-61.

27 Ruhiyyih Parris M, Chambers WA. Effects of the addition of potassium to prilocaine or bupivicaine. Br J Anaesth 1986; potassium to
$58: 297-300$.

28 Rouholamin E, Harris D. Axillary block anaesthesia in acute and elective hand surgery: a report on 300 procedures. Ann R Coll Surg Engl 1990;72:90-3. 